\title{
Optical bistability in two-dimensional nonlinear composites of coated cylinders with nonlinear core and graded shell
}

\author{
Ya M. Wu ${ }^{*}$, Guo Q. Chen, Wen C. Wu, Yi X. Wang, and Yang Huang ${ }^{* *}$ \\ School of Science, Jiangnan University, Wuxi 214122, PR China
}

Received: 10 January 2020 / Accepted: 21 September 2020

\begin{abstract}
The intrinsic optical bistability of the nonlinear composite consisting of coated cylinders with nonlinear core and graded Drude shell is investigated with self-consistent mean-field approximation. We derive the nonlinear equation of near field for such graded composite in the quasistatic limit. We demonstrate that the bistability threshold and the bistable width are highly dependent on the core radius, the incident frequency, and the graded coefficient of the coated cylinder inclusion. It is found that the optical bistability appears only when the core radius and the incident frequency satisfy some specific conditions. Therefore, the optical bistability for nonlinear composite materials may be optimized by the suitable adjustment of the physical and geometrical parameters. Our results may be helpful for the design of the nonlinear device with appropriate bistability.
\end{abstract}

Keywords: Optical bistability / cylindrical / graded particle / composite medium

\section{Introduction}

Optical bistability has attracted widespread interests for decades, due to its novel feature of controlling light with light [1-6]. For two-component nonlinear composite system, variational methods [7] and self-consistent mean-field approximation $[8,9]$ were developed to investigate the nonlinear response of composite system and to explore optical bistable behavior [7-9]. It is well-known that the local field enhancement of composite system may result in the optical bistability, which can be adjusted by controlling the local microstructure and the physical parameters of components in the composite system. With the development of nanotechnology, preparation of some special microstructures such as core-shell nanoparticle becomes possible [10-12]. For metal/semiconductor coreshell structure composite system, it was found that there exists large enhancement of the optical nonlinear susceptibility [13]. In addition, studies of nonlinear susceptibilities generated by two (three) harmonics in isotropic (or anisotropic) composite systems with core-shell nanoparticles were also widely investigated $[14,15]$. For instance, researchers found that the two-dimensional nonlinear composites exhibit huge enhancement of the third-order nonlinear optical susceptibility under the partial resonant condition [16,17]. Moreover, for the three-dimensional

\footnotetext{
* e-mail: wxwym2098@163.com

** e-mail: yanghuang@jiangnan.edu.cn
}

nonlinear composite consisting of the core-shell nanostructures, the nonlinearity of the whole composite system can be dramatically enhanced by adjusting the material properties of core-shell particle composition ratio $[18,19]$; Furthermore, Gao et al. [20,21] developed the self-consistent mean-field approximation to study the nonlinear composite media consisting of coated nanoparticles with nonlinear core and nonlinear shell. Recently, Shewamare et al. [22] studied the optical bistability of the random composites with nonlinear metallic nanoparticles. And Jackson et al. [23] showed that local field can get extremely high magnitude of Raman scattering enhancement in the order of $10^{6}$ in the nanoparticles with core-shell geometry.

On the other hand, effective linear dielectric constant [24], three-order nonlinear susceptibility [25] and two-(three) harmonics generations for the nonlinear susceptibilities [26] of the random granular composites with the graded core-shell structure were paid a lot of attentions. In this connection, Huang et al. [27,28] found that the gradient structure allows linear and nonlinear responses of the composite to show a wide plasmonic resonance band, which helps to enhance the effective nonlinear response of the composite. Later, Wei et al. [29] studied the local field distribution in nanoparticles with gradient shell and showed that the electric field in the shell is adjustable. More recently, third order nonlinear optical properties of magnetite nanoparticles in colloid were investigated experimentally as a sample of extension from electrical field to magnetic field [30].

To the best of our knowledge, the above works for graded composites only focus on the weak incident light 


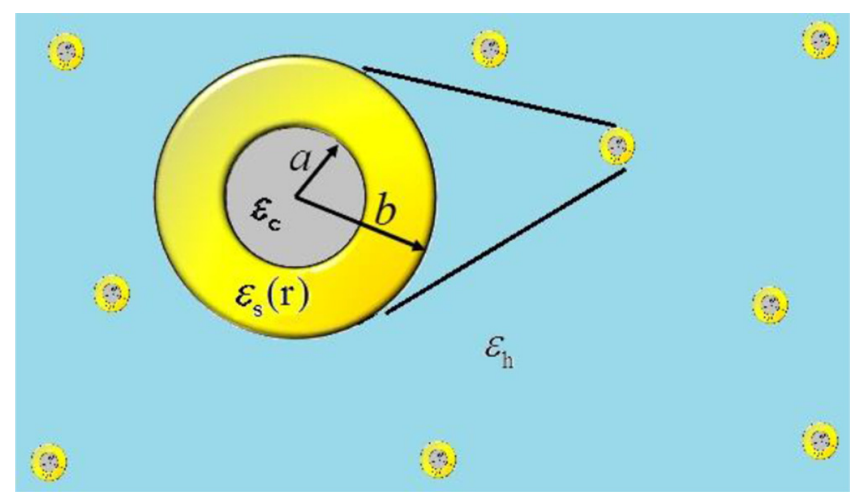

Fig. 1. Schematic diagram of the proposed composite in which the core-shell inclusions have Kerr-type dielectric cores and graded Drude-type shells.

intensity, and only the effective linear and nonlinear responses of the graded system were considered. It is well known that when the light intensity is strong, the composite system may exhibit optical bistability. Therefore, in this paper, we would like to investigate the optical bistable behavior of the graded composite media. Actually, under the surface plasmon resonant conditions, large enhancement of the local electric field takes place and results in the appreciable nonlinear bistability. With further enhancement of light intensity the combined the core-shell microstructure configuration and the gradient characteristic of cylinder components may provide us original optical bistable behavior. We believe our study may be helpful for the design and preparation of optical bistable materials and relative devices.

\section{Theoretical model}

We assume that the composite is composed of dilute coreshell cylinder inclusions randomly embedded in the linear host medium as shown in Figure 1 in 2D. The distance between inclusions is long enough to neglect the electrostatic dipole interactions among the inclusions in the dilute condition. The core-shell inclusions in this threecomponent composite consist of Kerr-type cores and graded Drude-type shells. Here, we make the assumption that the dielectric function of the graded materials varies along the radial direction $r$ in cylindrical coordinates $(r, \varphi, z)$, and can be written as $\varepsilon_{\alpha}(r)$ with the subscript $\alpha=c, s, h$ indicating the core, the shell, and the host media respectively. The inner and outer radii of the cylindrical shell are assumed to be $a$ and $b$. Within the quasi-static approximation, the electric potentials in whole space satisfy the following equation,

$$
\nabla \cdot\left[\varepsilon_{\alpha}(r) \nabla \phi_{\alpha}\right]=0,
$$

where $\phi_{\alpha}$ is electric potential in each area and corresponding local electric field is $\mathbf{E}_{\alpha}=-\nabla \phi_{\alpha}$. For infinitely long cylindrical configuration, Equation (1) reduces to

$$
\frac{1}{r} \frac{\partial}{\partial r}\left[\varepsilon_{\alpha}(r) r \frac{\partial \varphi_{\alpha}}{\partial r}\right]+\frac{1}{r} \frac{\partial}{\partial \phi}\left[\varepsilon_{\alpha}(r) \frac{\partial \varphi_{\alpha}}{r \partial \phi}\right]=0 .
$$

Therefore, the electric potentials in each region have the following general expressions,

$$
\begin{gathered}
\varphi_{c}(r, \phi)=-A r P_{1}(\cos \phi) E_{0}, \quad r \leq a, \\
\varphi_{s}(r, \phi)=\left[A_{1} R_{1}^{+}(r)+B_{1} R_{1}^{-}(r)\right] P_{1}(\cos \phi) E_{0}, \quad a<r \leq b,
\end{gathered}
$$

$$
\varphi_{h}(r, \phi)=\left(-r+b^{2} B r^{-1}\right) P_{1}(\cos \phi) E_{0}, \quad r>b,
$$

where $A, B, A_{1}, B_{1}$ are four unknown coefficients to be determined, $P_{m}(\cos \varphi)$ is the $m$-th order Legendre polynomials, and the radial function $R_{m}(r)$ in shell region satisfies the following equation

$$
\frac{1}{r} \frac{\partial}{\partial r}\left[\varepsilon_{s}(r) r \frac{\partial R_{m}(r)}{\partial r}\right]-\frac{m^{2}}{r^{2}}\left[\varepsilon_{s}(r) R_{m}(r)\right]=0, \quad a<r \leq b
$$

$R_{m}^{+}(r)$ and $R_{m}^{-}(r)$ are the two solutions which are regular at the origin and infinity, respectively. Applying the continuous boundary conditions of the normal components of the electric potentials and electric displacements, we can obtain the coefficients [29] as,

$$
\begin{gathered}
A=-a^{-1}\left[A_{1} R_{1}^{+}(a)+B_{1} R_{1}^{-}(a)\right], \\
B=[F(b)-1] /[F(b)+1], \\
A_{1}=2 b T_{2}^{-}(a) / T(a, b), \\
B_{1}=-2 b T_{2}^{+}(a) / T(a, b), \\
T(a, b)=T_{2}^{+}(a) T_{1}^{-}(b)-T_{2}^{-}(a) T_{1}^{+}(b), \\
T_{1}^{ \pm}(b)=R_{1}^{ \pm}(b)+b \frac{\varepsilon_{s}(b)}{\varepsilon_{h}} \frac{\partial}{\partial r} R_{1}^{ \pm}(b), \\
T_{2}^{ \pm}(a)=R_{1}^{ \pm}(a)-a \frac{\varepsilon_{s}(a)}{\varepsilon_{c}} \frac{\partial}{\partial r} R_{1}^{ \pm}(a), \\
F(b)=\frac{b \varepsilon_{s}(b)}{\varepsilon_{h} R_{1}(b)} \frac{\partial R_{1}(b)}{\partial r}, \\
R_{1}(r)=A_{1} R_{1}^{+}(r)+B_{1} R_{1}^{-}(r)
\end{gathered}
$$

where $B$ in equation (8) is equivalent to the dipole factor for the system and $F(b)$ in equation (14) is the equivalent dielectric constant of particles which are determined by the function of the gradation.

To further investigate the distribution of electric potentials in each region, we assume the cylindrical shell 
is described by the graded Drude model $[24,25]$

$$
\varepsilon_{s}(r, \omega)=1-\frac{\omega_{p}^{2}(r)}{\omega[\omega+i \Gamma]},
$$

where $\omega$ denotes the frequency of the external electric field, $\omega_{\mathrm{p}}(r)$ is the graded plasma frequency which varies spatially in radial direction and $\Gamma$ is the relaxation rate. Without loss of generality, we normalize the power-law-form graded plasma frequency as $\omega_{p}^{2}(r) / \omega_{p}^{2}=1-h r^{k}$ where $h$ and $k$ are two gradient constants denoting the gradation of the shell [31]. We further normalize the external field frequency $\omega$ and the relaxation rate $\Gamma$ with $\omega_{p}$, thus the Drude model can be rewritten as [29],

$$
\varepsilon_{s}(r, \omega)=1-\frac{\left(1-h r^{k}\right)}{\omega(\omega+i \Gamma)} .
$$

Substituting equation (17) into equation (6), we yield

$$
\begin{gathered}
R_{1}^{+}(r)=r F\left(\alpha_{+1}, \beta_{+1}, \gamma_{+1}, h r^{k} / d\right), \\
R_{1}^{-}(r)=r^{-1} F\left(\alpha_{-1}, \beta_{-1}, \gamma_{-1}, h r^{k} / d\right)
\end{gathered}
$$

where $F\left(\alpha, \beta, \gamma, h r^{k} / d\right)$ is the hypergeometric function which is analytic in the whole complex plane except at the singular points. In addition, $\alpha_{ \pm 1}=\left(1 \pm 2 / k-\sqrt{1+4 / k^{2}}\right) / 2$, $\beta_{ \pm 1}=\left(1 \pm 2 / k+\sqrt{1+4 / k^{2}}\right) / 2, \gamma_{ \pm 1}= \pm 2 / k+1$ and $d=$ $\omega^{2}+i \omega \Gamma-1$.

Therefore, we have analytically derived the potential solutions of graded cylindrical core-shell system with graded Drude model, hence it is easy to achieve the corresponding electric fields throughout the whole space with $\mathbf{E}_{\alpha}=-\nabla \phi_{\alpha}$

$$
\begin{gathered}
\left|E_{c}\right|=A E_{0}, \\
\left|E_{h}\right|=E_{0}\left[1+(b / r)^{4} B^{2}+2 B(b / r)^{2} \cos (2 \phi)\right]^{1 / 2} \\
\left|E_{s}\right|=E_{0}\left[\left(\partial R_{1}(r) / \partial r\right)^{2} \cos ^{2}(\phi)+R_{1}^{2}(r) r^{-2} \sin ^{2}(\phi)\right]^{1 / 2}
\end{gathered}
$$

In the dilute limit, we only consider the third-order nonlinear response of the core medium, in which the dielectric function of the core has a field-dependent expression as

$$
\tilde{\varepsilon}_{c}=\varepsilon_{c}+\chi_{c}\left|E_{c}\right|^{2},
$$

where $\chi_{\mathrm{c}}$ is the third-order nonlinear susceptibility. Substituting $\tilde{\varepsilon}_{c}$ for $\varepsilon_{c}$ in equation (7), we yield the nonlinear local field of core,

$$
E_{c}=-\nabla \varphi_{c}=-\nabla\left(-E_{0} \tilde{A} r \cos \theta\right)=\tilde{A} E_{0} .
$$

In the case of strong external field, the second term in equation (23) becomes comparable to the first term, so that the optical bistability in certain conditions may occur. Equation (24) is the key result of this work based on what we will give some numerical calculations and discussions in the following part.

\section{Numerical results and discussions}

We are now in a position to study the optical bistability in the graded composite materials. For numerical calculations, we choose the following physical parameters for the nonlinear core, graded shell, and host media: $\Gamma / \omega_{\mathrm{p}}=0.02$, $\varepsilon_{\mathrm{c}}=-10+0.037 i, \varepsilon_{\mathrm{h}}=64, \chi_{\mathrm{c}}=10^{-11}(\mathrm{~m} / \mathrm{V})^{2}[27-29]$. Note that the graded materials are rare in nature and most of them in study are artificial with some proper methods. As to the graded shell we prosed here, one possible way to achieve such gradation is to fabricate a graded metallic shell by using different noble metals as different layers covering the core. The large host dielectric constant is essential to achieve a bistable optical response. Such a large value of host medium may be realized by use of the $\mathrm{CuCl}$ material near the biexcitonic transition [32]. In reality, the dielectric constants of candidate materials who possess previous physical parameters vary with different frequency. Here we keep them constant for the sake of simplicity. Meanwhile, in the quasistatic approximation, the ratio of the inner and outer radii of the coated particle makes more sense than their exact value in the present model. Therefore, we keep the outer radius $b$ constant and provide it with a dimensionless value 1 , and vary the inner radius $a$ from 0 to 1 in the following calculation.

Figure 2a shows the local field in core as a function of the external electric field. It is evident that the S-shaped loop is found which indicates the optical bistable behavior in the near field with strong incident intensity. The abscissa $E_{0}$ of right and left inflection points in the bistable curve are, respectively, the upper and lower thresholds. Figure 2a illustrates that the upper and lower thresholds will simultaneously vary with the core radius $a$. It is found that both the upper and lower thresholds are increased with increasing the core radius in the small value regime, and then are decreased. Moreover, for some specific values of $a$, for instance $a=0.31$, the bistable threshold has the maximal width with $4.584 \times 10^{6} \mathrm{~V} / \mathrm{m}$ as shown in Figure 2b; When $a=0.93$ however, the optical bistable curves will disappear. This indicates a critical value for core radius $a_{\mathrm{c}}$ in the determination of optical bistablity within the present physical parameters, i.e., when the particle core radius $a<a_{c}$, the bistability appears; The bistability would disappear if $a>a_{c}$. Besides that, dash line in Figure 2b shows the dependence of upper and lower thresholds on the core radius for the non-graded shell $(h=0)$. Variation of bistable thresholds with $a$ in the non-graded case is quite different from that in the graded case. In details, there exists a certain value of $a$ after which the upper and lower thresholds will decrease in the graded case. In non-graded case however, the upper and lower thresholds vary slowly with $a$, but finally increase rapidly when $a$ is close to 1 . Note that all the optical bistability occur under strong field 

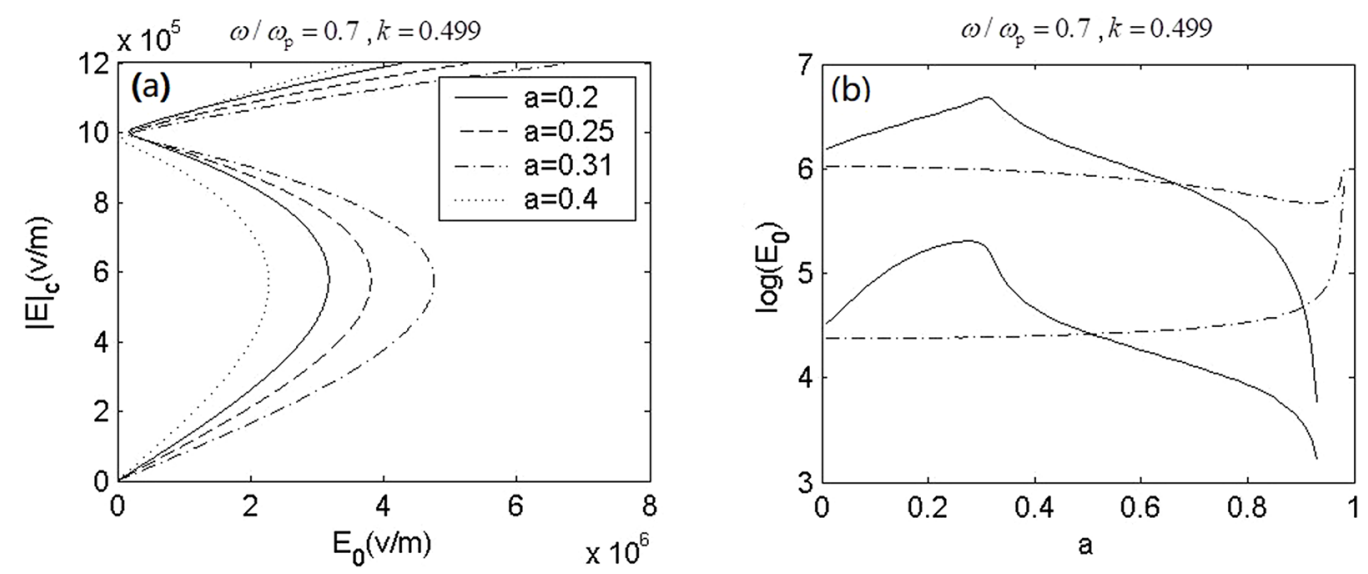

Fig. 2. (a) The local electric field as a function of the external field with different core radii. (b) The upper and lower thresholds as a function of core radius $a$ with $h=0.9$ (solid line) and $h=0$ (dashed line), respectively.
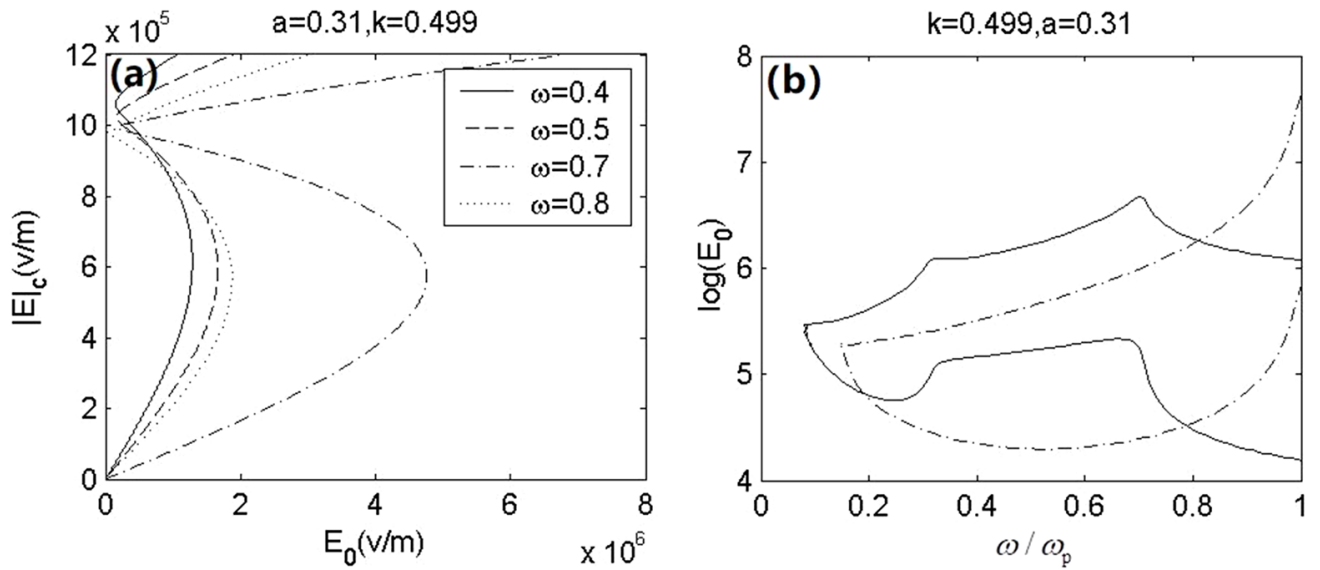

Fig. 3. (a) The local electric field as a function of the external field with different frequency $\omega / \omega_{\mathrm{p}}$. (b) The upper and lower thresholds as a function of frequency $\omega / \omega_{\mathrm{p}}$ with $h=0.9$ (solid line) and $h=0$ (dashed line), respectively.

intensity condition and one could not achieve this bistable curve with weak field intensity. These results provide us a good theoretical guidance for the design of low-threshold optical biastability.

Figure 3a shows the local electric field as a function of the external field with different frequency $\omega$. The maximal width of the threshold is $4.584 \times 10^{6} \mathrm{~V} / \mathrm{m}$ which occurs at $\omega / \omega_{\mathrm{p}}=0.7$. Once $\omega / \omega_{\mathrm{p}}$ is down to 0.08 , the upper and lower threshold curves begin to degenerate and finally the optical bistability disappear when $\omega / \omega_{\mathrm{p}}<0.08$. This indicates that there is a critical incident frequency $\omega_{\mathrm{c}}$, above which bistability appears and below which the bistability disappers. This may result from the fact that the average local field of core is weaker when $\omega<\omega_{\mathrm{c}}$, so the bistablity does not appear. With the increase of $\omega$, the local field also increases, hence leading to bistable phenomenon. Compared to the gradient case $(h=0.9)$ composite with non-gradient media $(h=0)$ can achieve lower thresholds and larger width of optical bistability thresholds.
To illustrate the dependence of upper and lower thresholds on $a$ and $\omega / \omega_{\mathrm{p}}$ in a boarder parameter space, we define the width of bistable threshold $\Delta E_{0}$ and plot it in Figure 4. Again, the width of bistable threshold is found tunable by adjusting the core radius $a$ and external field frequency $\omega / \omega_{\mathrm{p}}$. When $a=0.01$ and $\omega / \omega_{\mathrm{p}}=0.97$, the maximal width of threshold is found to be $1.121 \times 10^{7}(\mathrm{~V} / \mathrm{m})$. Thus, it is possible to achieve the optimal width of threshold by our suitable adjustment of $a$ and $\omega / \omega_{\mathrm{p}}$ simultaneously.

Figure 5 illustrates bistable curves in near field with different host media, it is obviously found that the upper threshold reduces when the value of $\varepsilon_{\mathrm{h}}$ increases. To further demonstrate, we investigate the dependence of thresholds on the host media $\varepsilon_{\mathrm{h}}$ in Figure 6 . It shows that the upper and lower thresholds are simultaneously reducing with increasing $\varepsilon_{\mathrm{h}}$, especially in the low $\varepsilon_{\mathrm{h}}$ regime, i.e., from 1 to 4. After that, they vary slowly with $\varepsilon_{\mathrm{h}}$ and are almost unchanged when $\varepsilon_{\mathrm{h}}$ reaches to high values. The situation is quite different in the non-graded case (dashed line) that the 


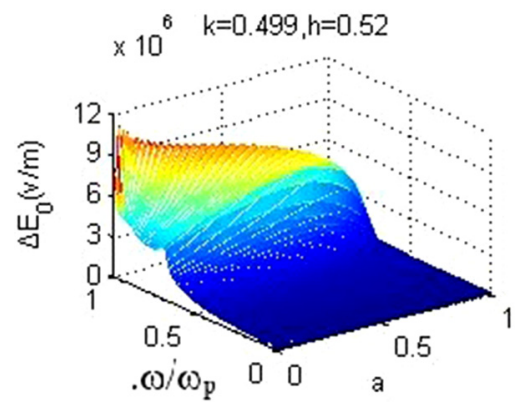

Fig. 4. The width of bistable threshold as a function of $a$ and $\omega / \omega_{\mathrm{p}}$

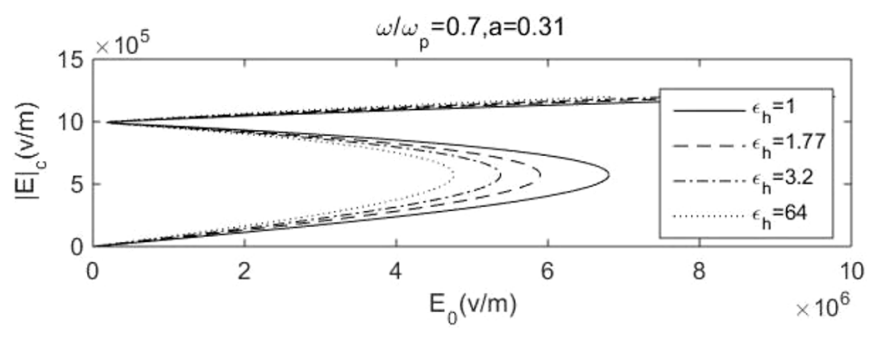

Fig. 5. The local field as a function of the external field with different host media. The gradation constant $h=0.9$.

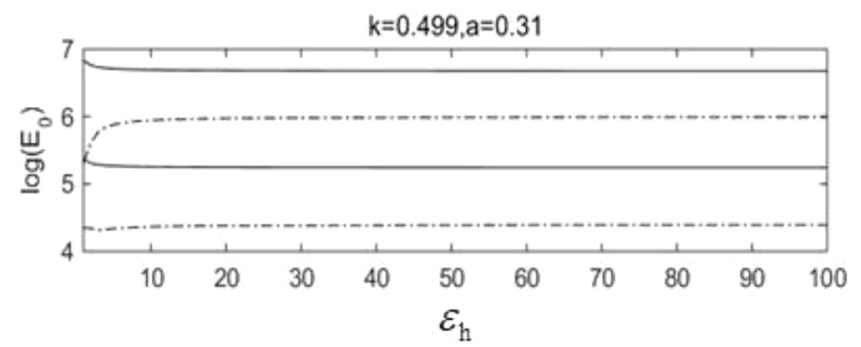

Fig. 6. The upper and lower thresholds as a function of host medium with $h=0.9$ (solid) and $h=0$ (dashed).

upper threshold is increasing rapidly in the low $\varepsilon_{\mathrm{h}}$ regime from 1 to 6 , while the lower thresholds firstly reduce and then slightly increase. In the high $\varepsilon_{\mathrm{h}}$ regime, the dependence of upper and lower thresholds on $\varepsilon_{\mathrm{h}}$ is similar to the graded case.

In the end, we study the dependence of the upper and lower thresholds on the gradient factor $h$. It can be seen in Figure 7 that there are two peaks for the upper thresholds. The first peak occurs at $h=0.52$ that corresponds to the width of threshold of $5.637 \times 10^{6} \mathrm{~V} / \mathrm{m}$, and the second happens at $h=0.9$ with the width of threshold being $4.584 \times 10^{6} \mathrm{~V} / \mathrm{m}$. When $h$ continues to increase, both the upper and lower thresholds exhibit a downward trend.

Our results suggest that there exists the optical bistability under certain conditions for cylindrical composite consisting of nonlinear coated nanocylinders. This nonlinear feature mainly results from the surface plasmon resonance (SPR) inside the nanostructure, which can

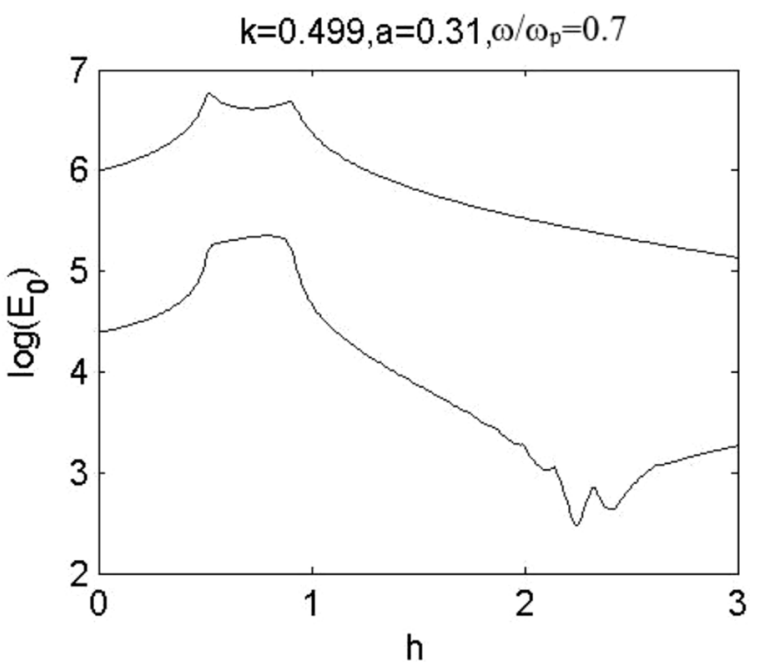

Fig. 7. The upper and lower thresholds as a function of $h$.

dramatically enhance the local electric field inside the nonlinear core, hence boosting the nonlinear response. Therefore the optical bistability occurs. Actually, the SPR enhanced optical nonlinearity resulting in the optical bistability in composite media are the conclusions demonstrated in many previous studies [33-35]. In addition, the introduction of gradient shell give a new degree of freedom for us to obtain desired optical bistability threshold. In the end, we shall remark that although all the parameters in the present manuscript might be realized experimentally, it still has many challenges in precisely preparing the proposed model and testing the bistable behavior in experiment.

\section{Conclusion}

Within quasistatic approximation, we derive the nonlinear equations of near field for the graded metal-dielectric composite, which is composed of Kerr dielectric coregraded metallic shell nano-cylinders randomly embedded in a linear host medium, and study the optical bistability in such composite. We demonstrate the dependences of the bistability threshold and width on the core radius, the frequency, and gradient coefficient. There exist a critical core radius $a_{\mathrm{c}}$ and a critical incident frequency $\omega_{\mathrm{c}}$, below (above) which the optical bistable behavior takes place. The results indicate that the gradient shell structure is in favor of appearance of optical bistability with lower threshold and larger width of threshold. Moreover, the character of optical bistability can be tunable by changing the geometrical and physical parameters of composite, to achieve maximal width of threshold and lower thresholds. The results can provide a theoretical basis for the design and preparation of optical bistable material.

This work was supported by the National Natural Science Foundation of China (grant no. 11704158) and the Natural Science Foundation of Jiangsu Province (grant no. BK20170170). 


\section{References}

1. K.M. Leung, Phys. Rev. A 33, 2461 (1986)

2. R.W. Boyd, Nonlinear optics (New York: Academic, 1992)

3. H.M. Gibbs, S.L. McCall, T.N.C. Venkatesan, Phys. Rev. Lett. 36, 113 (1976)

4. H. Chang, H.B. Wu, C.D. Xie, H. Wang, Phys. Rev. Lett. 93, 213901 (2004)

5. T.K. Mavrogordatos, et al., Phys. Rev. Lett. 118, 040402 (2017)

6. M. Kim, S. Kim, S. Kim, Opt. Express 26, 11620 (2018)

7. D.J. Bergman, O. Levy, D. Stroud, Phys. Rev. B 49, 129 (1994)

8. L. Gao, L.P. Gu, Z.Y. Li, Phys. Rev. E 68, 066601 (2003)

9. Y. Huang, L. Gao, Opt. Lett. 43, 2836 (2018)

10. N.A. Nicorovici, R.C. McPhedran, G.W. Miton, Phys. Rev. B 49, 8479 (1994)

11. K. Mallik, M. Mandal, N. Pradhan, T. Pal, Nano. Lett. 1, 319 (2001)

12. J.J. Schneider, Adv. Mater. 13, 259 (2001)

13. A.E. Neeves, M.H. Birnboim, J. Opt. Soc. Am. B 6 (1989)

14. P.M. Hui, C. Xu, D. Stroud, Phys. Rev. B 69, 014203 (2004)

15. X.C. Jiang, Y.W. Zhou, D.L. Gao, Y. Huang, L. Gao, Opt. Express 28, 17384 (2020)

16. O. Levy, J. Appl. Phys. 77, 1696 (1995)

17. L. Gao, Phys. Status Solidi B 236, 182 (2003)
18. X.Y. Liu, Z.Y. Li, Phys. Lett. A 223, 475 (1996)

19. C. Xu, Z.Y. Li, Phys. Lett. A 252, 233 (1999)

20. L. Gao, Phys. Lett. A 318, 119 (2003)

21. L.P. Gu, L. Gao, Physica B 368, 275 (2005)

22. S. Shewamare, V.N. Mal'nev, Physica B 407, 4837 (2012)

23. J.B. Jackson, S.L. Westcott, L.R. Hirsch, J.L. West, N.J. Halas, Appl. Phys. Lett. 82, 257 (2003)

24. J.P. Huang, K.W. Yu, G.Q. Gu, M. Karttunen, Phys. Rev. E 67, $054105(2003)$

25. L. Gao, J.P. Huang, K.W. Yu, Phys. Rev. B 69, 075105 (2004)

26. L. Gao, K.W. Yu, Phys. Rev. B 72, 075111 (2005)

27. J.P. Huang, K.W. Yu, J. Opt. Soc. Am. B 22, 1641 (2005)

28. J.P. Huang, P.M. Hui, K.W. Yu, Phys. Lett. A 342, 484 (2005)

29. E.B. Wei, Chin. Phys. B 19, 107802 (2010)

30. D.H.G. Espinosa, C.L.P. Oliveira, A.M.F. Neto, J. Opt. Soc. Am. B 35, 346 (2018)

31. J.P. Huang, K.W. Yu, Phys. Rep. 431, 87 (2006)

32. K.L. Stokes, A. Puri, Opt. Lett. 15, 986 (1990)

33. J.W. Haus et al., J. Opt. Soc. Am. B 6, 797 (1989)

34. N. Kalyaniwalla, J.W. Haus, R. Inguva, M.H. Bimboim, Phys. Rev. A 42, 5613 (1990)

35. D.J. Bergman, O. Levy, D. Stround, Phys. Rev. B 49, 129 (1994)

Cite this article as: Ya M. Wu, Guo Q. Chen, Wen C. Wu, Yi X. Wang, Yang Huang, Optical bistability in two-dimensional nonlinear composites of coated cylinders with nonlinear core and graded shell, EPJ Appl. Metamat. 7, 6 (2020) 